\title{
Role of the tip vortex in the force generation of low-aspect-ratio normal flat plates
}

\author{
MATTHEW J. RINGUETTE $\dagger$ MICHELE MILANO \\ AND MORTEZA GHARIB \\ Graduate Aeronautical Laboratories, California Institute of Technology, \\ 1200 E. California Blvd, Pasadena, CA 91125, USA
}

(Received 26 May 2005 and in revised form 18 January 2007)

We investigate experimentally the force generated by the unsteady vortex formation of low-aspect-ratio normal flat plates with one end free. The objective of this study is to determine the role of the free end, or tip, vortex. Understanding this simple case provides insight into flapping-wing propulsion, which involves the unsteady motion of low-aspect-ratio appendages. As a simple model of a propulsive half-stroke, we consider a rectangular normal flat plate undergoing a translating start-up motion in a towing tank. Digital particle image velocimetry is used to measure multiple perpendicular sections of the flow velocity and vorticity, in order to correlate vortex circulation with the measured plate force. The three-dimensional wake structure is captured using flow visualization. We show that the tip vortex produces a significant maximum in the plate force. Suppressing its formation results in a force minimum. Comparing plates of aspect ratio six and two, the flow is similar in terms of absolute distance from the tip, but evolves faster for aspect ratio two. The plate drag coefficient increases with decreasing aspect ratio.

\section{Introduction}

Work on the flapping-wing propulsion of insects and birds has increased greatly in recent years, motivated by its potential application to the design of micro air vehicles. However, there is a corresponding lack of fundamental research on unsteady threedimensional vortex flows at the appropriate low to moderate Reynolds numbers. The present study focuses on understanding the effect of the wingtip vortex on the overall wing force of a hovering insect. This tip effect is dependent on the wing aspect ratio $(A R)$.

Flying insects have wing $A R$ s between 2.75 and 6 (Ellington 1984; Dickinson, Lehmann \& Sane 1999), while hummingbird wings have ARs of about 4 (Dhawan 1988). Aspect ratio is defined here as $b^{2} / S$, where $b$ is the span of a single wing and $S$ is the single-wing planform (top-view) area. Hovering wing kinematics typically include a segment of downward, plunging motion which tilts the drag force upward so that it contributes to supporting the insect's weight; for dragonflies and hoverflies, drag is the primary wing force keeping the insect aloft (Wang 2004). Here, we focus on drag-based hovering.

$\dagger$ Present address: Department of Mechanical and Aerospace Engineering, Princeton University, Princeton, New Jersey 08544, USA.

$\ddagger$ Present address: Department of Mechanical and Aerospace Engineering, Arizona State University, Tempe, Arizona 85287, USA. 
A hovering wing attains high angles of attack and produces unsteady force through the vortices generated by flow separation at its leading and trailing edges, as well as at its tip. Suction from the leading-edge vortex (LEV) has been shown to be partly responsible for the wing force generated during hovering (Dickinson \& Götz 1993; Ellington et al. 1996). However, the function of the tip vortex (TV) is disputed in the literature (Birch \& Dickinson 2001), and the force production capability of the TV has not been studied.

We investigate the role of the TV by using a model with simplified kinematics and geometry that isolates the tip effect. This effect is uncovered by varying the relative strength of the TV through changing the model's $A R$ and tip, or end, condition. We consider a low- $A R$ flat plate of rectangular planform with one end, the tip, free; the rectangular wing allows a clear distinction between the vortex generated at the tip and those produced at the two leading edges. The plate angle of attack is set at $90^{\circ}$, so that the plate force is primarily drag. An insect wing maintains a nearly constant angle of attack throughout the majority of each half-stroke, making the fixed angle of attack approximation reasonable (Dickinson \& Götz 1993). The plate motion is a rectilinear translation starting from rest, which approximates a hovering half-stroke. This simple configuration results in a flow that is surprisingly complex, but nonetheless amenable to analysis.

There are few studies on the unsteady starting flow of normal flat plates, and virtually none that consider low- $A R$ flat plates having a free end condition. Normal flat plate starting motion with nominally two-dimensional flow conditions was investigated experimentally by Sarpkaya \& Kline (1982), Lian \& Huang (1989), Dennis et al. (1993) and Chua et al. (1990), who also performed computations. Koumoutsakos \& Shiels (1996) presented two-dimensional viscous computations that confirmed the existence of an instability in the shear layers emanating from the plate's edges; the numerical simulation of Luchini \& Tognaccini (2002) showed a similar instability. An analytical model to determine the force on a two-dimensional accelerating flat plate at fixed angles of attack was developed by Pullin \& Wang (2004) and applied to hovering insect flight. The model agreed well with numerical simulations of a hovering insect wing and identified unsteady vortex lift and added mass as hovering mechanisms.

Lisoski (1993) investigated the force on normal flat plates accelerating from rest in a towing tank to Reynolds numbers of order 1000, with end conditions designed to ensure primarily two-dimensional flow. For plates of $A R$ between 6 and 17, he measured a minimum in the drag force after the initial acceleration peak. This minimum was not observed for a plate of $A R=10$ with one end free, indicating the significant effect of the tip.

For the present work, the effect of the TV is investigated by measuring the drag on plates of different $A R$ and varying the tip condition. Digital particle image velocimetry (DPIV) is used to measure multiple chordwise and spanwise sections of the flow velocity, and to obtain vorticity and circulation data for the two LEVs and the TV. These data on vortex formation and strength are related to the measured plate force. Flow visualization is used to obtain the three-dimensional flow structure.

\section{Experimental setup}

Figure 1 shows a schematic of the experimental set-up. The experiments were conducted in a glass-walled towing tank of interior dimensions $450 \mathrm{~cm} \times 96 \mathrm{~cm} \times$ $78 \mathrm{~cm}$, with water as the working fluid. A servomotor-driven carriage moves above 


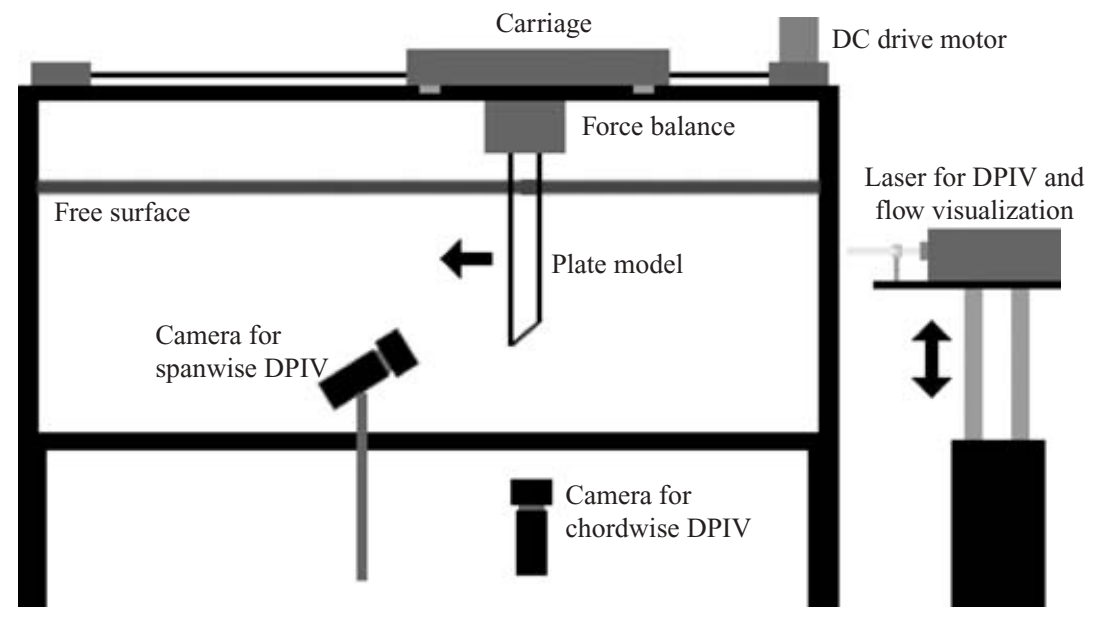

FIGURE 1. Schematic of the towing tank and DPIV hardware.

the tank on rails parallel to the longest sides. Attached below the carriage is a force balance, to which the flat-plate models are secured so that they are submerged vertically in the tank. Unsteady carriage velocity profiles are achieved using a programmable position controller.

Two plate $A R \mathrm{~s}, 6$ and 2, were tested; the $A R$ was set by adjusting the water level. The plate velocity profile was a constant acceleration from rest over a distance of $1 / 4$ of the plate chord, followed by a constant velocity, $U_{\text {final }}$, with a total travel of about 8 chord lengths. This motion is similar to that of an insect wing during the downstroke (Dickinson \& Götz 1993; Dickinson et al. 1999). We chose a Reynolds number of 3000 , which is within the $10^{2}-10^{4}$ range of flying insects (Dudley 2000). Here, the Reynolds number is defined as $R e=c U_{\text {final }} / v$, where $c$ is the plate chord length and $v$ is the kinematic viscosity of water.

The flat-plate model for DPIV was made from $0.32 \mathrm{~cm}$ thick window glass with $c=6.35 \mathrm{~cm}$; it was sufficiently rigid and cast no disruptive shadow in the camera image. A stiffer, lighter model, constructed from a $0.34 \mathrm{~cm}$ thick unidirectional carbon fibre composite with $c=5 \mathrm{~cm}$, was used for the force measurements. Each edge of the carbon fibre model was bevelled at a $30^{\circ}$ angle, while the edges of the glass model were left square. The inherent edge shear-layer instability was observed for both edge geometries.

Force measurements were performed using a strain gauge force balance with a static resolution of $\pm 0.0005 \mathrm{~N}$, equating to resolutions of \pm 0.02 and \pm 0.06 in the drag coefficients for $A R=6$ and 2, respectively (for more details see Lisoski 1993). The strain gauge amplifier output was low-pass filtered in hardware and software, using cutoff frequencies of 5 and $2 \mathrm{~Hz}$, respectively, to remove the $4-10 \mathrm{~Hz}$ noise from the towing tank drive system. For each aspect ratio, 10 runs were averaged to produce the final force trace.

We used DPIV to capture two-dimensional sections of the flow velocity quantitatively. This technique employs cross-correlation to obtain velocity fields from the displacement of particles between two images separated in time (see Willert \& Gharib 1991). The DPIV data were taken in horizontal (chordwise) planes at the 50,75 and $90 \%$ spanwise locations (measured from the free surface), as well as in vertical (spanwise) planes, parallel to the direction of travel, at mid-chord and at one of the leading edges. 

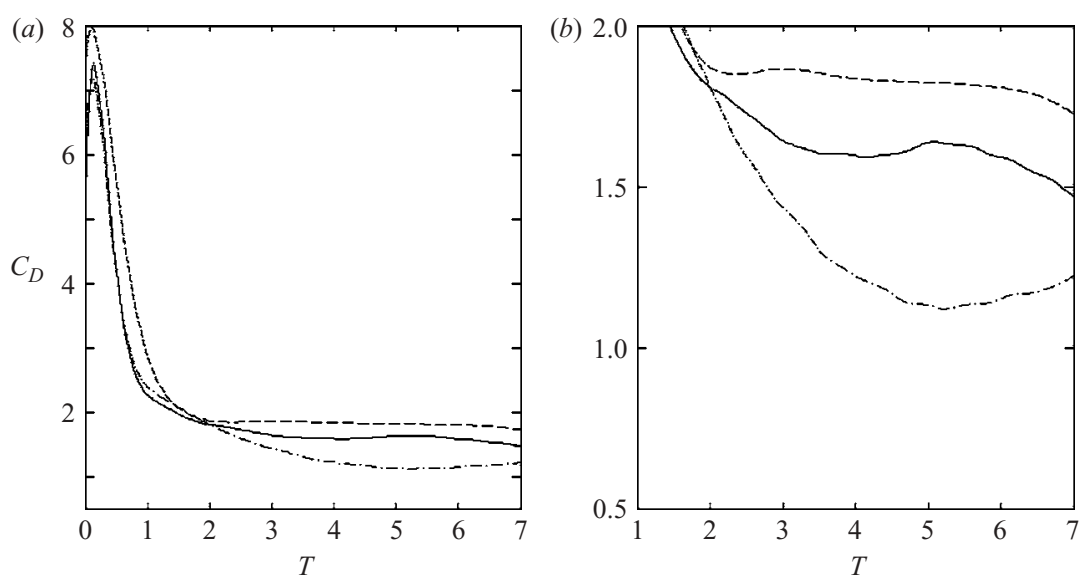

Figure 2. (a) $C_{D}$ for $A R=6$ and 2 plates during a start-up translation, as a function of $T$ (see main text for definition). (b) Detail of $(a)$. Both $(a)$ and $(b)$ show $C_{D}$ for the free tip $A R=6$ plate (continuous line) and for the same plate with the tip grazing a raised bottom (dash-dotted line); the dashed line is the $C_{D}$ for the $A R=2$ plate with the tip free.

For DPIV, the towing tank was seeded with neutrally buoyant silicon-coated hollow glass spheres approximately $10 \mu \mathrm{m}$ in diameter, which were illuminated using a pulsed $\mathrm{Nd}$ :YAG laser spread into a thin sheet. A $30 \mathrm{~Hz}$ CCD video camera with a resolution of $768 \times 480$ pixels captured the particle images. The camera was placed below and to the side of the tank to image the chordwise and spanwise flow sections, respectively (see figure 1). We used the laser/camera timing technique given in Willert \& Gharib (1997) to achieve the required $5 \mathrm{~ms}$ time steps between pairs of particle images.

The DPIV processing was done as described by Willert \& Gharib (1991) using a $32 \times$ 32 pixel interrogation window with a $16 \times 16$ pixel overlap, which gave 1440 vectors for each velocity field. A typical camera field of view was about $35 \mathrm{~cm} \times 25 \mathrm{~cm}$, resulting in a spatial resolution of about $0.7 \mathrm{~cm} \times 0.8 \mathrm{~cm}$; the uncertainty in the velocity and vorticity measurement is $\pm 1 \%$ and $\pm 3 \%$, respectively. The measurement noise was reduced using an interrogation window-shifting technique given in Westerweel, Dabiri \& Gharib (1997). An entire run did not fit within the camera field of view, so two DPIV measurements, with an overlap of about $2 c$, were taken and composited to capture a single run.

The instability in the edge shear layers was under-resolved by the DPIV measurements, creating high-frequency 'jitter' in the vortex circulation calculated from the DPIV-derived vorticity field, $\omega(\boldsymbol{x}, t)$. The following low-pass filter, having a cutoff frequency of $0.66 \mathrm{~Hz}$, was used to remove this noise from $\omega(\boldsymbol{x}, t): \omega_{f i t e r}\left(\boldsymbol{x}, t_{k+1}\right)=$ $0.8 \omega_{\text {filter }}\left(\boldsymbol{x}, t_{k}\right)+0.2 \omega\left(\boldsymbol{x}, t_{k+1}\right)$.

The DPIV position and velocity data were normalized using $c$ and $U_{\text {final }}$, respectively. Results are plotted versus a non-dimensional time, $T$, defined as $T=t \bar{U} / c$, where $\bar{U}=\left(1 / t \int_{0}^{t} U(\tau) \mathrm{d} \tau\right)$ is the running mean of the plate velocity, $U$. This quantity is approximately equal to the number of chord lengths the plate has travelled.

\section{Results}

\subsection{Drag force measurements}

Figure 2 gives the drag coefficient, $C_{D}$, versus time for $A R$ of 6 and 2; figure $2(b)$ highlights significant features near $T=5$. The drag coefficient is computed using the 
measured drag, $D, U_{\text {final }}$, the submerged plate area, $S$, and the density of water, $\rho$, as follows: $C_{D}=2 D / \rho U_{\text {final }}^{2} S$. Figure $2(a)$ shows a peak in the $C_{D}$ at the start-up for each case, due primarily to added mass and the inertia of the plate model. For the $A R=6$ free-end case (solid line), the most prominent feature of the data after the initial drag peak is a broad maximum centred on $T=5$ (figure $2 b$ ).

To uncover the effect of the plate's free end, or tip, on the drag force, the $A R=6$ runs were repeated with a raised bottom wall placed less than $1 \mathrm{~mm}$ below the plate's free end, in order to suppress the flow around the tip. This 'grazing' end condition, coupled with a clean free surface at the upper end, was recommended by Slaouti \& Gerrard (1981) and Lisoski (1993) for achieving predominantly two-dimensional flow along the span of a model in a towing tank. For this experiment (plotted as a dash-dotted line in figure 2), there is instead a $C_{D}$ minimum centred on $T=5$. The corresponding free-end maximum is $46 \%$ greater.

Lisoski (1993) also reported drag minimums, or 'buckets', for $A R$ from 6 to 17 with the grazing lower-end condition. He attributed the phenomenon to a symmetrical recirculating wake bubble, which breaks up at about 10 chord lengths of travel. For the free-end case, he measured a drag bucket for $A R=17$, but no minimum for $A R=10$. This demonstrates that the effect of the tip becomes significant for $A R$ nearing 10 , and the present study shows that a substantial gain in drag force is achieved when the $A R$ is reduced further to 6 .

For the $A R=2$ plate with a free tip (figure 2, dashed line) the drag coefficient is greater than that of $A R=6$ after $T=2$. This is due to the relatively larger tip effect of the $A R=2$ plate, as described in $\S 3.3$.

\subsection{Chordwise flow sections}

To determine the effect of the tip on the vortex dynamics along the span, DPIV was used to capture chordwise sections of the flow velocity and vorticity at the 50, 75 and $90 \%$ spanwise locations, measured from the free surface. The flow in the chordwise planes is characterized by separation and roll-up into vortices at the two leading edges, and is symmetrical about the mid-chord location. We calculate the circulation of the LEVs using Stokes' theorem in two dimensions, $\Gamma=\int_{A} \omega \mathrm{d} A$, where $\Gamma$ is the in-plane circulation, $\omega$ is the out-of-plane vorticity, and $A$ is the area enclosing it. A Matlab program tracks the LEV core locations, determines their areas based on a vorticity threshold, and computes both the LEV circulation and the total circulation produced at the edges. The circulation is averaged about the wake symmetry-line and is non-dimensional (see $\S 2$ ).

Figure 3(a) shows the total and LEV circulation versus $T$ for $A R=6$ at each spanwise location. At $90 \%$ span, the LEVs remain attached to the plate throughout the run, so that the total and LEV circulation coincide. After $T=0.5$, the total circulation is progressively lower with decreasing distance from the tip. The circulation of the LEVs in each section eventually reaches a maximum value and saturates, but this saturation time decreases with decreasing distance from the tip. Therefore, the effect of the tip is to limit the strength and growth of the vortices nearest it.

Vorticity fields at 50 and $75 \%$ span are given as insets on the corresponding circulation plots in figures 4 and 5, respectively; the flow at $90 \%$ span is reported in figure 7. All vorticity fields are presented using iso-vorticity contours with a nondimensional range of $-40-40$ and a contour interval of 1 ; dashed contour lines indicate negative vorticity.

Figure 4 shows that the saturation of the LEV circulation at $50 \%$ span corresponds to the start of the LEV pinch-off process, which is completed at $T=6.67$. At $75 \%$ 

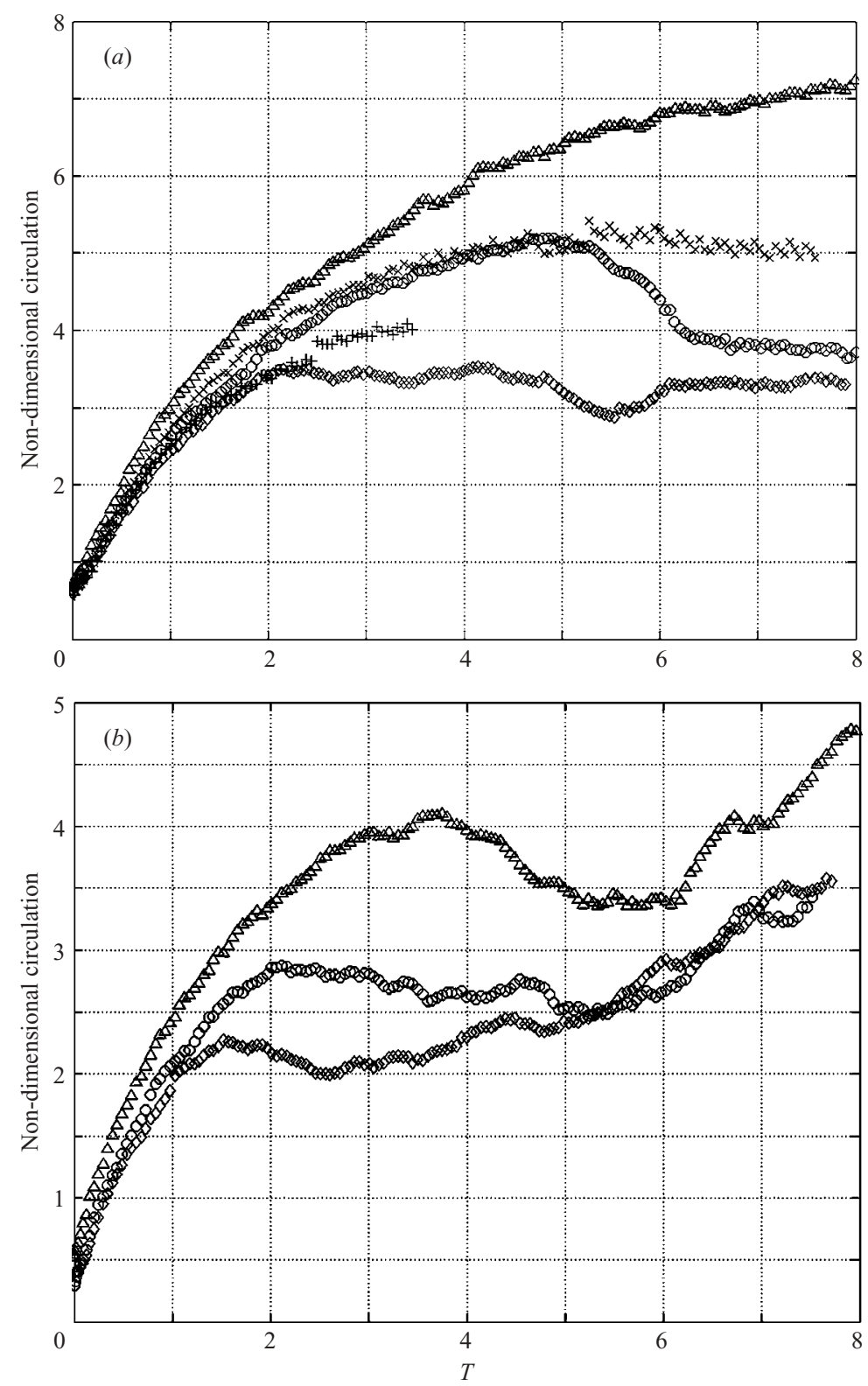

Figure 3. Circulation $v$ s. $T$ for $(a) A R=6$ and $(b) A R=2 . \triangle$, total circulation, $50 \%$ span; $\times$, LEV circulation, $50 \%$ span; $\bigcirc$, total circulation, $75 \%$ span; + , LEV circulation, $75 \%$ span; $\diamond$, total circulation, $90 \%$ span.

span, a near-pinch-off occurs at the saturation time, $T=3.1$, after which the LEVs merge again with the edge shear layers (figure 5). In contrast to the data at 50 and $90 \%$ span, the total circulation at $75 \%$ span reaches a maximum at $T=5$, then drops and levels off. This loss in sectional circulation is probably due to predominantly threedimensional out-of-plane velocity affecting the DPIV measurement. After $T=5$, the LEVs are drawn toward the centre-wake and undergo a momentary, 'inward' pinch-off. 


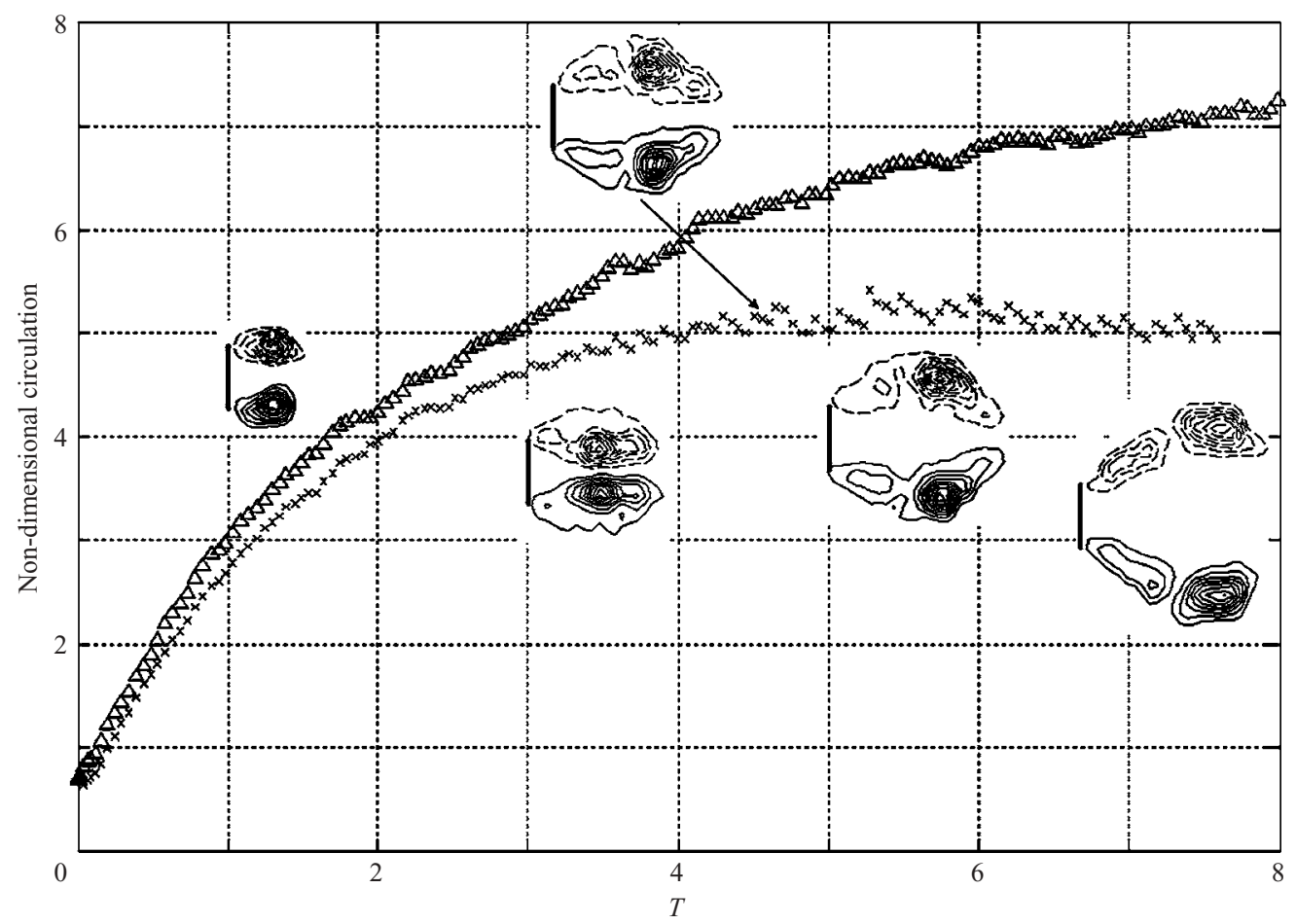

FiguRE 4. Circulation at $A R=6,50 \%$ span, with vorticity field insets. $\triangle$, total circulation; $\times$, LEV circulation. The plate locations correspond to the appropriate time, $T$, unless otherwise indicated.

Section 3.3 shows that this behaviour is the result of highly three-dimensional flow induced by the tip vortex.

Curves of the total circulation at 50,75 and $90 \%$ span for $A R=2$ are shown in figure 3(b); for this $A R$, the total and LEV circulation coincide. The most prominent feature of these data is that, for $T<6$, the total circulation at $A R=2,50 \%$ span is qualitatively similar to that of $A R=6$ at $75 \%$ span, whereas the $A R=2,75 \%$ span case resembles that of $A R=6,90 \%$ span. The sections in each similar pair are located at nearly the same absolute distance from the plate tip (the same plate is used for both $A R$ s). This suggests that the flow at each $A R$ develops in a similar way spatially, which is reflected in the vorticity data. Vorticity field snapshots for $A R=2,50 \%$ span show that an 'inward' pinch-off of the LEVs takes place after the maximum in total circulation (figure 6), which is also observed for the $A R=6,75 \%$ span case. However, this event occurs at an earlier time for $A R=2$, indicating that the flow evolves more quickly; this is the result of its relatively greater tip effect.

\subsection{Spanwise and chordwise flow sections compared to measured drag}

Both the spanwise and chordwise vorticity fields are given together as insets on plots of the drag force measurements in figures 7 and 8. Spanwise sections of the flow velocity were measured at mid-chord (centre-wake) and at one of the leading edges, in planes parallel to the direction of travel. In each vorticity inset, all five sectional vorticity fields are arranged in a 'tiled' format, with the three chordwise cases displayed 


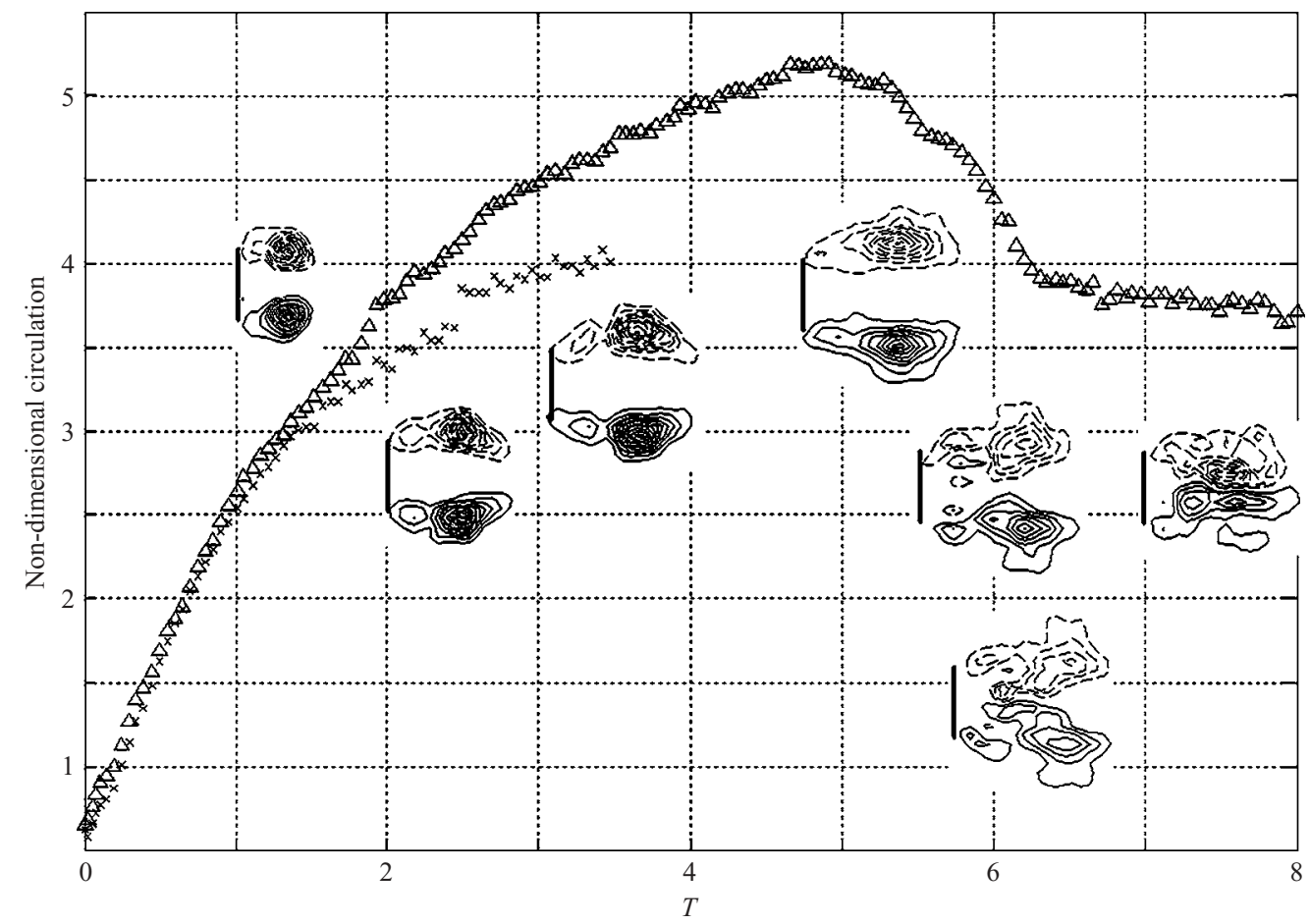

FIGURE 5. Circulation at $A R=6,75 \%$ span, with vorticity field insets. $\triangle$, total circulation; $\times$, LEV circulation. The plate locations correspond to the appropriate time, $T$.

in descending order on the left-hand side, the mid-chord case in the middle, and the leading-edge case on the right. The vorticity fields in each inset, for a specific aspect ratio, are plotted at the same length scale.

The measured drag and vorticity fields for $A R=6$ are given in figure 7. At $T=2$, the tip vorticity in the mid-chord plane starts an apparent upward growth along the span. Flow-visualization images given in $\S 3.4$ show that this new vorticity has its source at the plate's corners. The change in the chordwise wake structure at $90 \%$ span corresponds to this spanwise vorticity crossing that location (figure $7 a$ ).

After the drag maximum at $T=5$, the iso-vorticity contours in the spanwise plane at mid-chord form a 'neck' at the $75 \%$ span level (figure $7 b, T=5.73$ ). The vorticity insets at $T=5.73$ and 6.67 show that this behaviour is the start of an 'upward' pinch off process, where the accumulated vorticity above the TV at mid-chord pinches off from the TV itself. This event coincides with the 'inward' pinch-off of the chordwise LEVs at $75 \%$ span, suggesting that the $75 \%$ spanwise location marks the transition between tip- and leading-edge-dominated flow.

The vorticity data reveal two flow phenomena responsible for the $A R=6$ drag maximum centred on $T=5$. The first is the accumulation of attached vorticity near the tip, apparent in the mid-chord plane, which reaches its maximum strength at $T=5$ (before the 'upward' pinch-off). This vorticity creates a low-pressure region on the leeward face of the plate, thus increasing the drag. The second is the effect of the strong LEVs at $50 \%$ span. The force, $F$, due to the LEVs can be described by assuming that they are point vortices and using the following relationship (the 


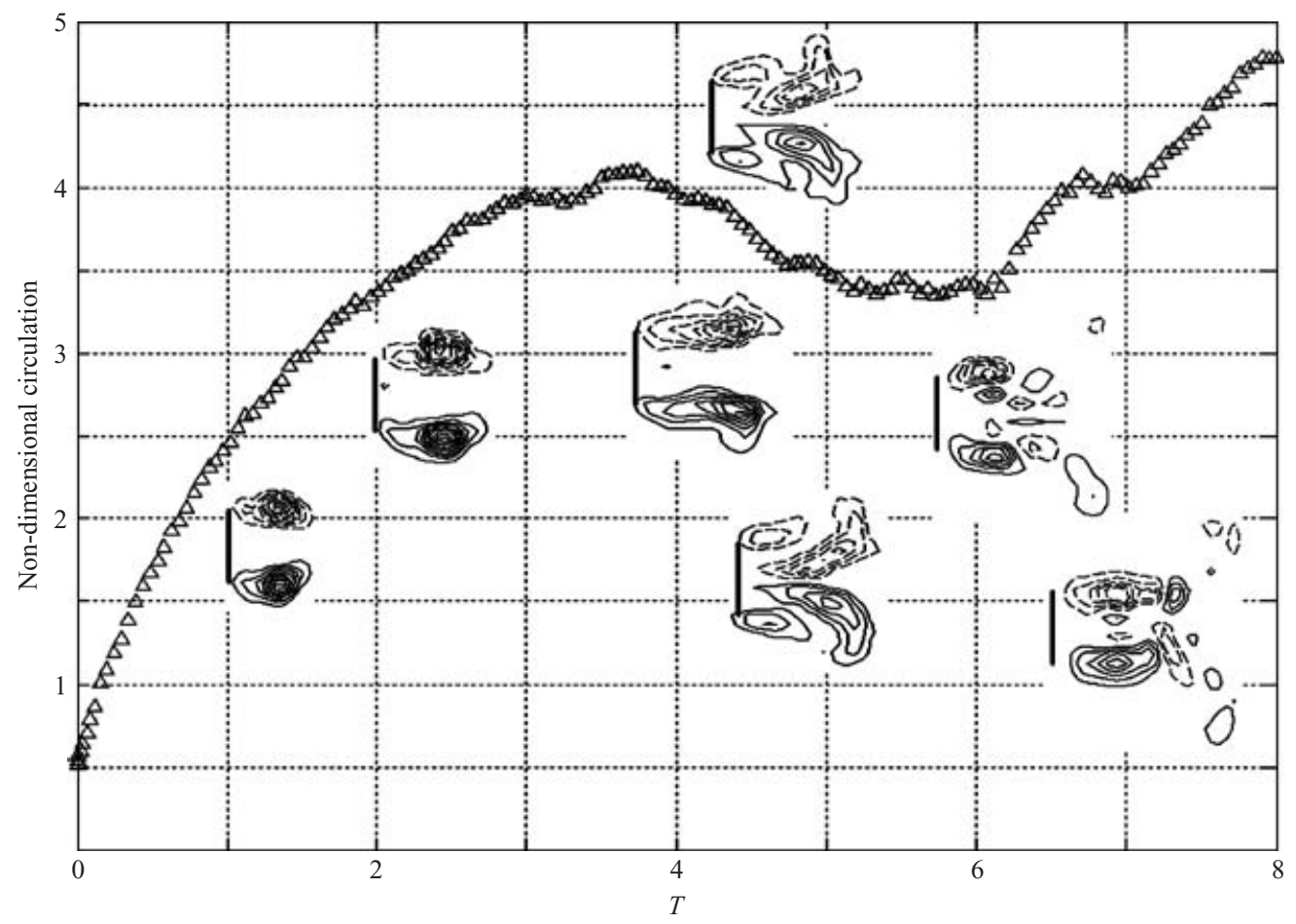

FIgURE 6. Circulation at $A R=2,50 \%$ span, with vorticity field insets. $\triangle$, total circulation. The plate locations correspond to the appropriate time, $T$.

quantity in square brackets is the impulse) (Batchelor 1967):

$$
F \propto \frac{\mathrm{d}}{\mathrm{d} t}\left[-\left(\sum_{i} Y_{i} \Gamma_{i}\right) \hat{\boldsymbol{i}}+\left(\sum_{i} X_{i} \Gamma_{i}\right) \hat{\boldsymbol{j}}\right]
$$

the assumption of two-dimensional flow is reasonable until after the LEVs pinch off. Considering the chordwise plane at $50 \%$ span, the direction of the $x$ unit vector, $\hat{i}$, is taken as opposite that of the plate motion (i.e. right), with the origin located at mid-chord, so that $\hat{j}$ is directed upward. Each LEV (subscript $i$ ) has its circulation, $\Gamma_{i}$, concentrated at $\left(X_{i}, Y_{i}\right)$, which is the centroid of the vortex. The formula indicates that, after the circulation saturates, the drag force increases with the rate that the LEVs move away from the wake centreline. At $50 \%$ span, the chordwise vorticity data show that this rate peaks shortly after $T=5$, then drops following the pinch-off of the LEVs at $T=6.67$.

Plots showing the measured drag for $A R=2$ along with tiled vorticity field insets are given in figure 8 . Consistent with the chordwise data, the spanwise flows of $A R=2$ and 6 are similar spatially, in terms of absolute distance from the tip. The same 'necking' of the mid-chord plane vorticity that is observed for $A R=6,75 \%$ span also occurs for $A R=2,50 \%$ span (figure $8 b, T=4.39$ ). However, the flow in the $A R=2$ spanwise datasets evolves faster, which is in agreement with the chordwise vorticity. The 'upward' pinch-off of mid-chord plane vorticity and 'inward' pinch-off of the LEVs at $75 \%$ span take place at $T=5.73$ for $A R=6$ (figure $7 b$ ), whereas the same events for $A R=2$ occur earlier at $T=4.39$ (figure $8 b$ ). 


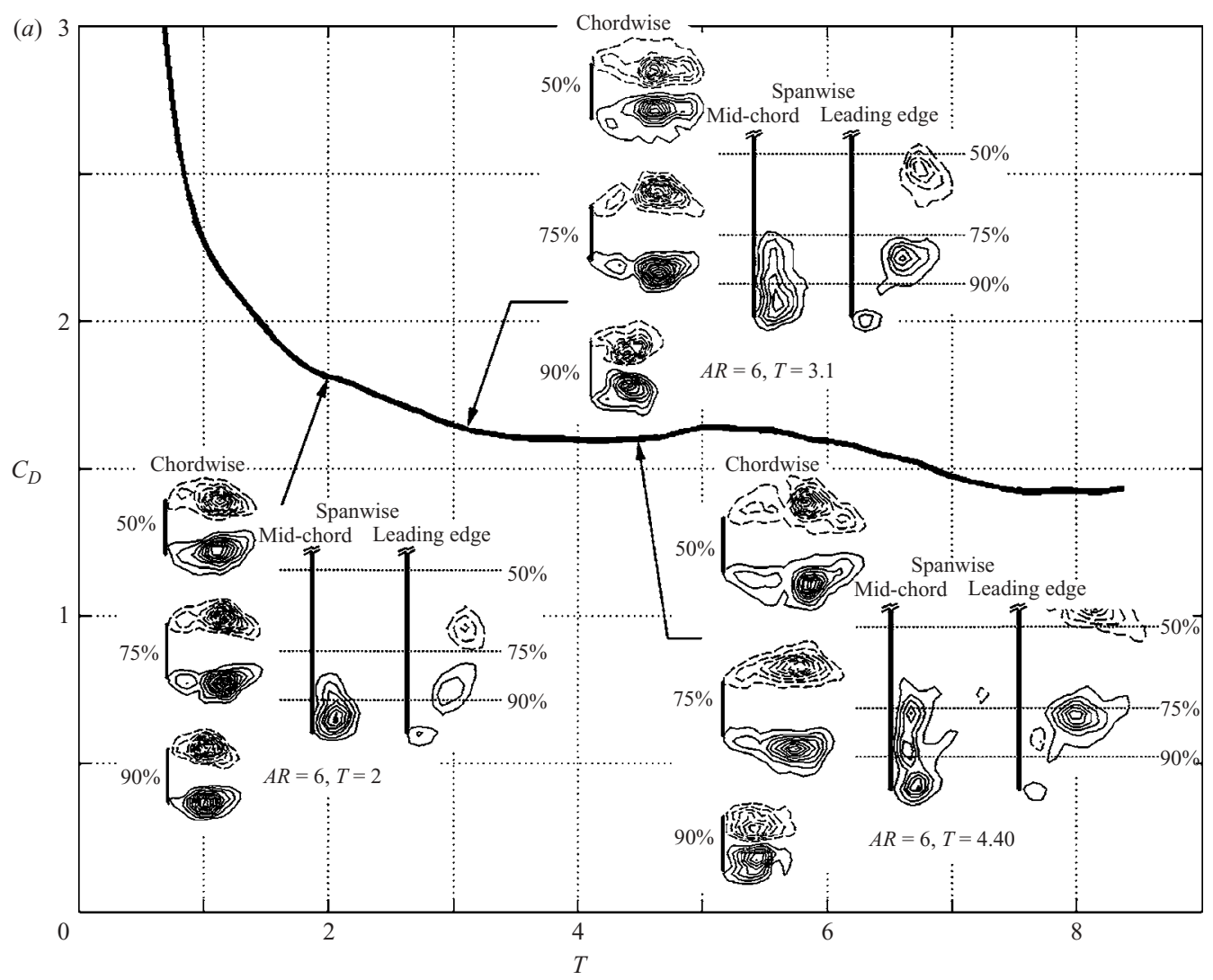

Figure $7(a)$. For caption see facing page.

It follows that the drag coefficient for $A R=2$ is greater than that of $A R=6$, after $T=2$, because of its relatively stronger tip effect. The low-pressure region created by the tip vortex and the accumulation of attached vorticity above it (observed in the mid-chord plane) affect a greater portion of the span for $A R=2$.

\subsection{Structure of the flow}

We model the structure of the flow near the tip at the start-up using vortex lines to represent the LEVs and the TV. From induced velocity arguments and flow visualization, we create a sequence of snapshots showing how the vortex lines evolve (figure 9). The snapshots give an isometric, leeward view of the right-hand corner of the plate tip; thin lines represent the vortex lines and arrows show their sense of rotation. During acceleration from rest, the flow separates at the leading and tip edges, and rolls up to form vortices behind each edge; these vortices connect in the corner (figure $9 a$ ). As the LEV and the TV grow stronger, they induce a velocity that acts to rotate the corner vortex line away from the plate surface (figure $9 b$ ). Once this vortex is lifted off the plate, the induced velocities from the 'upright' legs of its horseshoe-like structure cause the legs to 'fold' toward one another (figure $9 c$ ). The induced velocities from the folded portions of each leg act to push the vortex line connecting them back toward the plate face. Each end of this connecting line then 


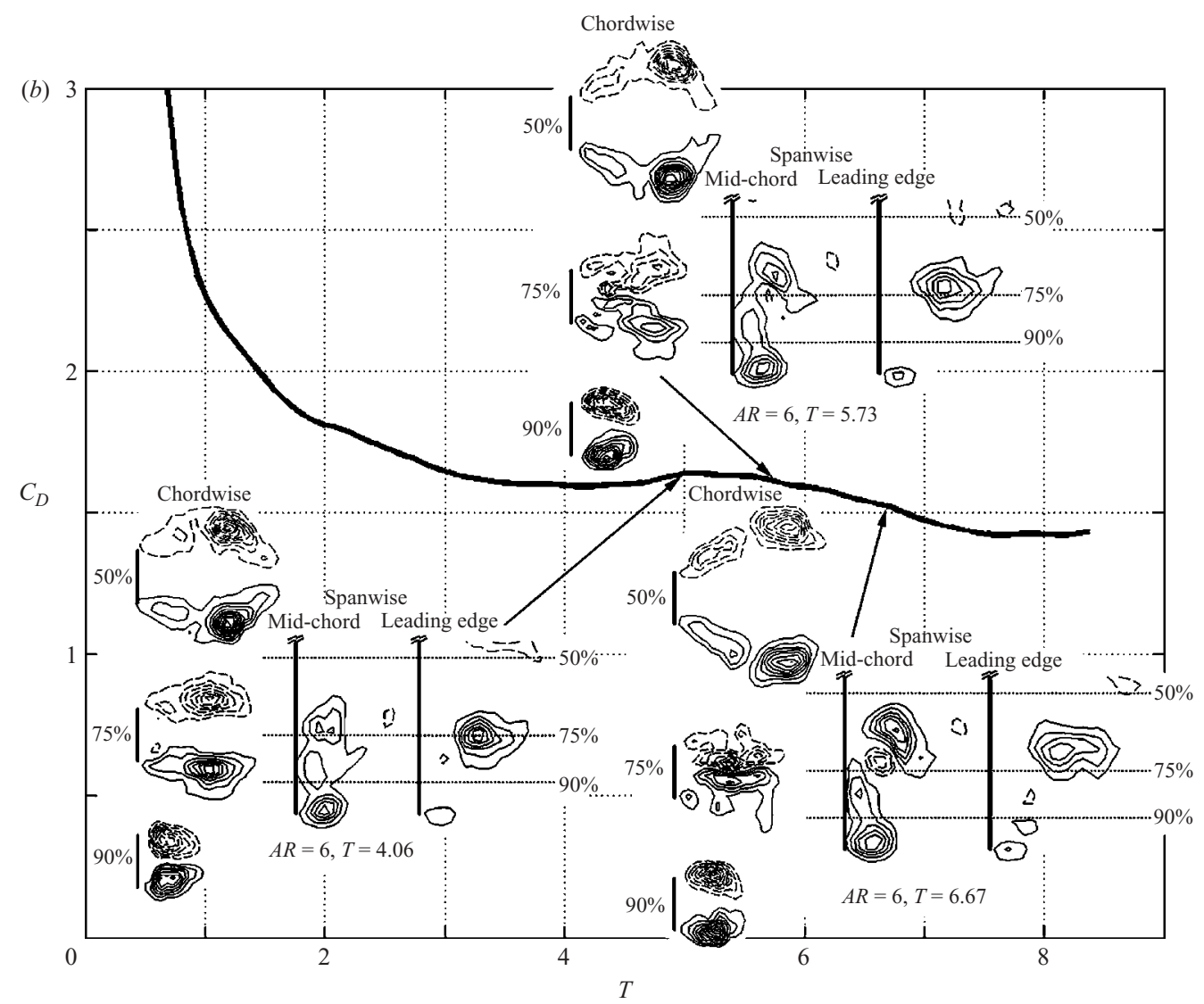

FIgURE 7. Measured $C_{D} v s . T$ for $A R=6$, including tiled vorticity insets showing the flow at significant times.

kinks and forms an inverted ' $\mathrm{V}$ ' with the leg next to it (figure 9d); adjacent legs of each V have opposite rotational directions. From flow visualization in the plate corners, it is clear that the velocity of the inner legs of each V, i.e. those closest to the plate corner, is greater than that of the outer legs. This leads the outer legs to twist around their respective inner legs, following the rotational velocities of the inner legs, forming vortex loops (figure $9 e$ ). The twisting of each looped vortex perpetuates into a double helix pattern, whose rotational sense is given by the centrelines in figure $9(e-f)$.

The large-scale three-dimensional flow structure of the $A R=6$ plate was imaged using fluorescent dye injected from two rakes attached to the upstream face of the plate in the tip region. A laser cone was used for illumination, while a CCD video camera captured the images. Figure 10(a) gives a snapshot from this visualization, taken after the plate has travelled 2.6 chord lengths. The picture shows an isometric view of the lower-half of the plate; the visualization was done at $R e=2000$ to yield clearer images. Dye was fed into the wake via the edge shear layers, creating an absence of dye at the leeward plate surface. The instability in the edge shear layers, described by Koumoutsakos \& Shiels (1996), is visible as vertically oriented ripples enclosing the wake. Note that the band of dye stretching diagonally from the left of 


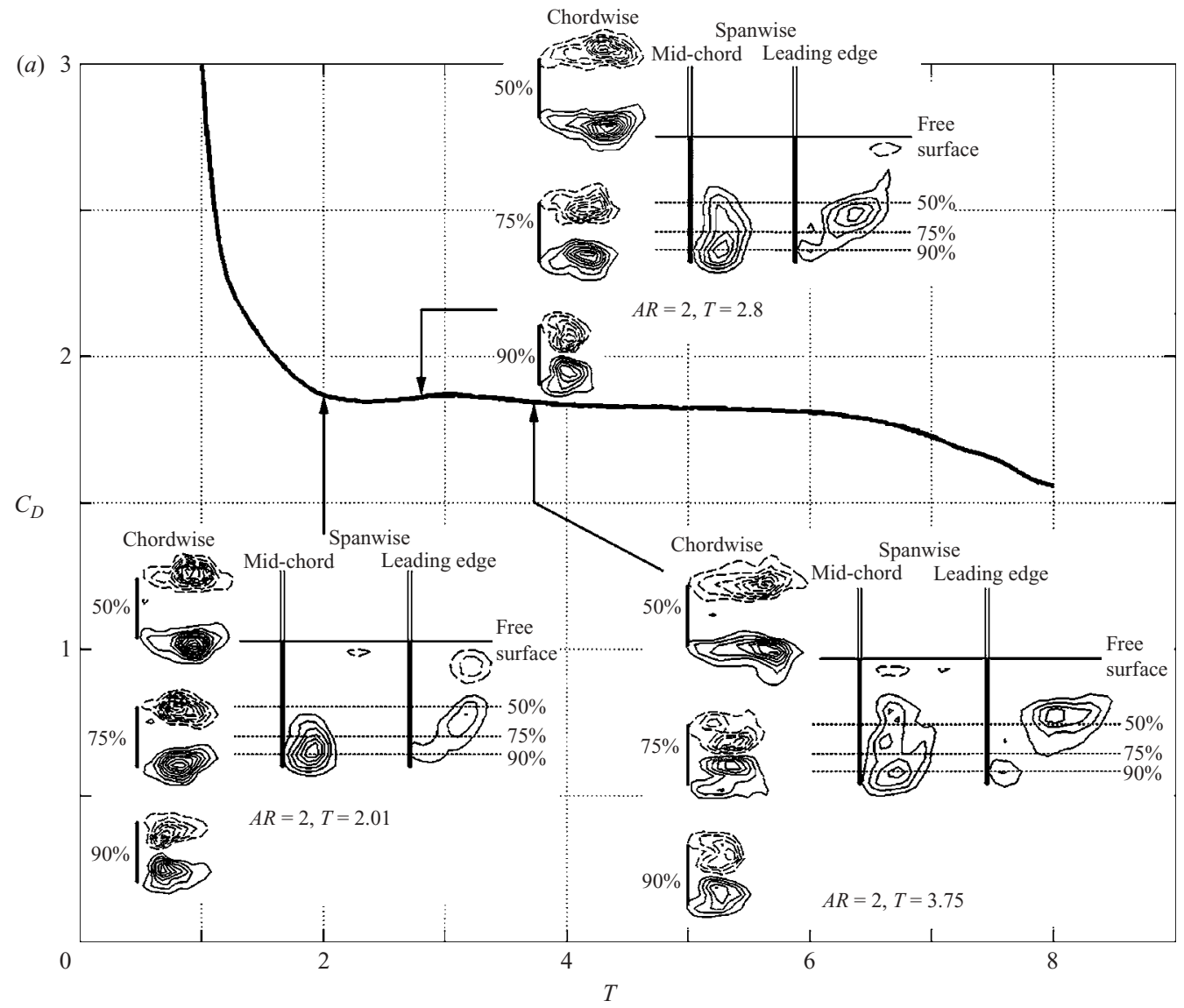

Figure $8(a)$. For caption see facing page.

the image to the plate is not part of the LEVs, but is simply being convected between them toward the plate.

The visualization movie shows that the LEVs near the tip remain closely attached to the plate, whereas the LEVs above the tip grow farther from the plate with time; this is consistent with the DPIV measurements. The DPIV data show that the LEVs away from the tip are stronger, indicating that they have comparatively lower pressure than those closer to the tip. This pressure gradient causes a spanwise flow within the LEVs that is directed away from the tip, which is observed in the movie as an upward progression of the dye marking the LEVs. This upward flow within the LEVs is also reflected in the DPIV velocity data. Figure $10(b)$ shows an instantaneous velocity field obtained from DPIV in a vertical plane located at the leading edge and oriented parallel to the flow; the plate $A R$ is 6 with $R e=3000$, and the total plate travel is also 2.6c. The measurement plane intersects the inner portion of the LEV at that edge, so that the velocity vectors above the tip are primarily directed at the back of the plate. The upward flow velocity within the LEV is evident above a distance of about 2 chord lengths from the tip, consistent with the dye visualization. This spanwise flow velocity within the LEVs, coupled with their rotational velocity, gives them a somewhat helical structure, which is best seen in the left-most LEV in figure 10(a). It is also apparent 


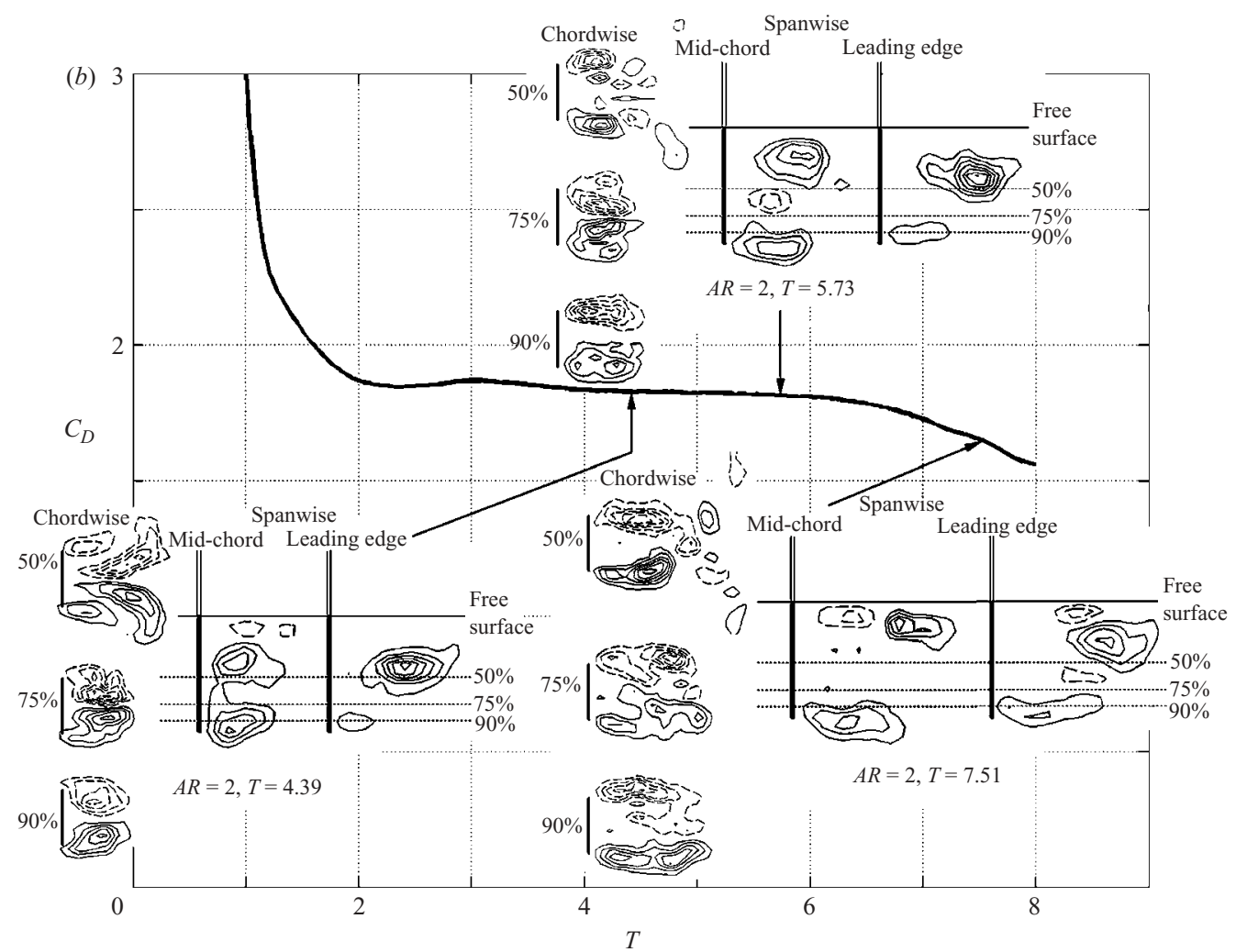

Figure 8. Measured $C_{D}$ vs. $T$ for $A R=2$, including tiled vorticity insets showing the flow at significant times.

from the dye snapshot that the tip effect bends the LEVs so that their vorticity vectors have components perpendicular to the leading-edge plane, which explains the existence of vortices in the spanwise DPIV data at the leading edge.

A feature common to both the $A R=2$ and 6 cases is the rapid increase in vorticity above the TV in the mid-chord plane, occurring between $T=2$ and 4 (see $\S 3.3$ ). We tested whether the corner vortices are the source of this vorticity, rather than an upward growth of the TV. A small drop of fluorescent dye was 'painted' in each corner of the leeward face of the plate, and a spanwise laser sheet, parallel to the flow, was placed at mid-chord to illuminate any dye convected there. Figure 11 gives three snapshots from this experiment, which was done at $R e=3000$ with an $A R=6$ plate. The image in figure 11(a), taken at three chord lengths of travel, shows that some of the dye has been drawn into the TV. Snapshots taken at 0.27 and $0.33 \mathrm{~s}$ later, respectively, show a substantial amount of dye introduced above the TV. This dye has its source at the plate corners, so it is likely that the accumulation of vorticity in the mid-chord plane is due to vorticity from the corners convected there by the LEVs.

\section{Concluding remarks}

The role of the tip vortex in the unsteady force-generation of low-aspect-ratio flat plates with one end free is investigated experimentally. We consider the case 

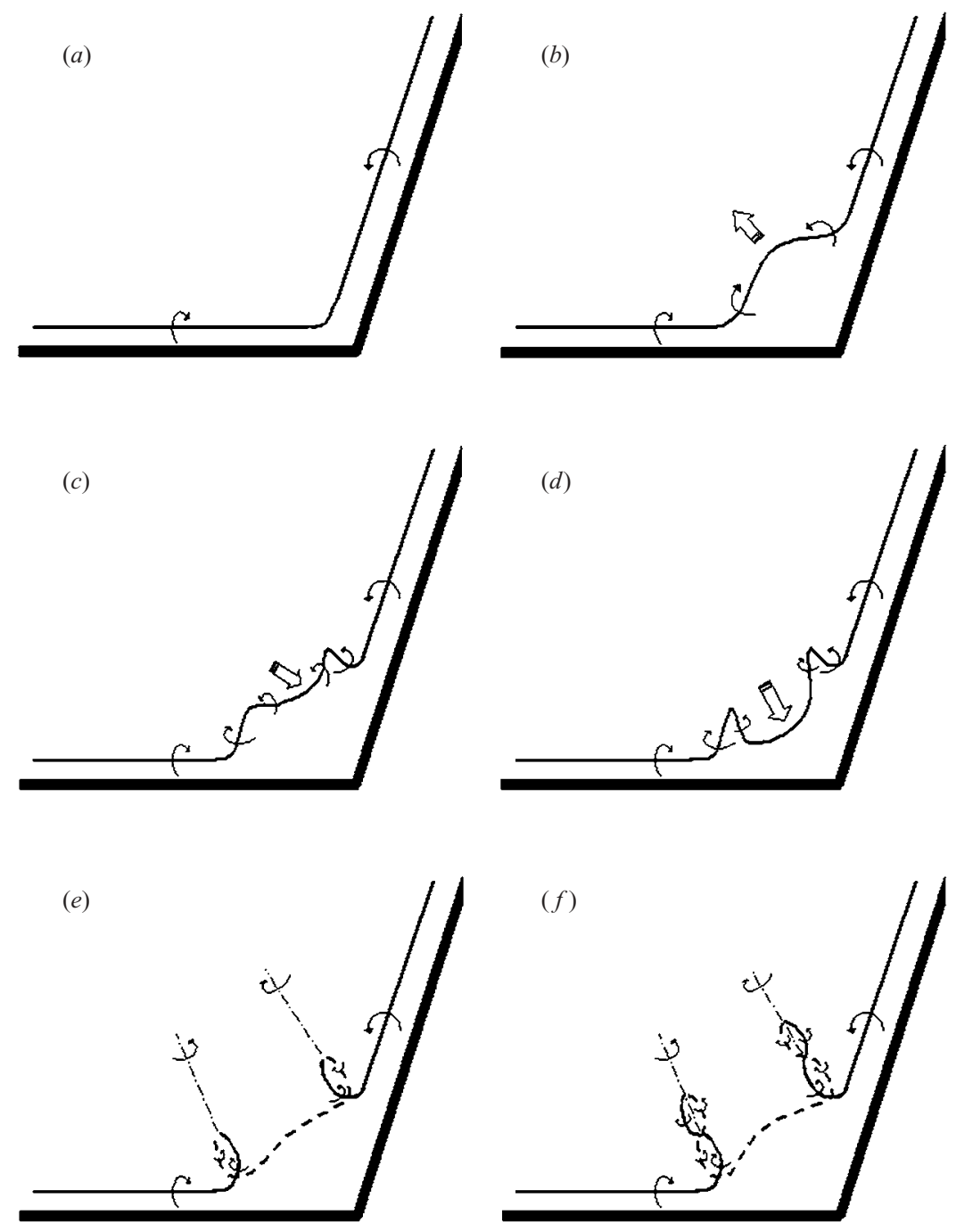

FIGURE 9. Vortex line model of the flow near the tip at the start-up. View of the right-hand corner of the leeward plate face; the flow is symmetrical about the mid-chord line.

of a rectangular normal flat plate undergoing a translating starting motion akin to the propulsive half-stroke of an insect wing. We measure the plate drag and use DPIV to capture multiple, perpendicular sections of the flow velocity. For the range of wing aspect ratios and stroke amplitudes found in nature, we find that the tip vortex contributes substantially to the overall plate force. The results show that the interaction between the tip and leading-edge vortices generates a high transient drag peak, whereas suppressing the flow around the tip results in significantly lower drag. To date, studies of insect flight have not considered the force production capability of the tip vortex. The findings of the present investigation suggest that work on the role of the tip vortex for more complicated flapping motions and wing geometries is warranted. 
(a)
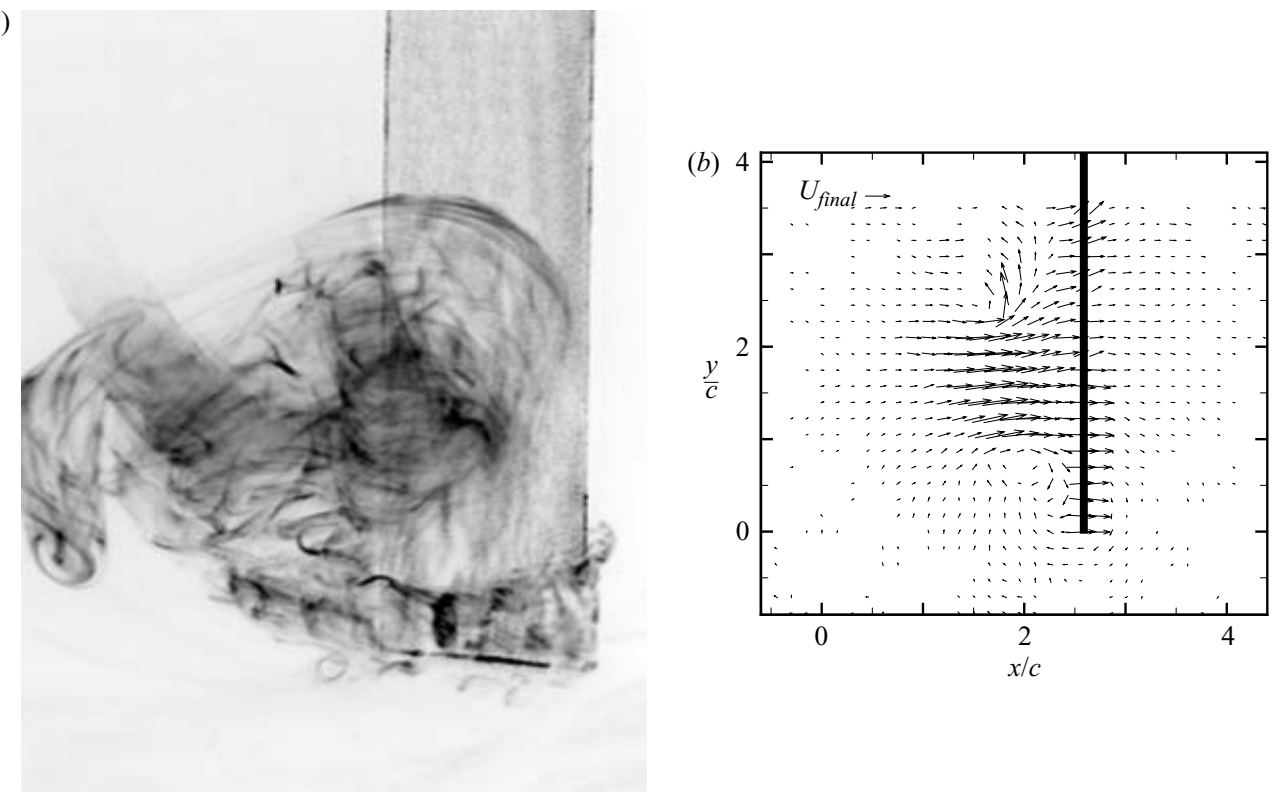

Figure 10. (a) Three-dimensional dye visualization at plate travel of 2.6c. $R e=2000, A R=6$. The plate is travelling right; dye is injected from rakes upstream near the tip. $(b)$ Velocity field from DPIV in a vertical plane at the leading edge; total plate travel is $2.6 c$. $R e=3000$, $A R=6$; the measurement plane is parallel to the flow.
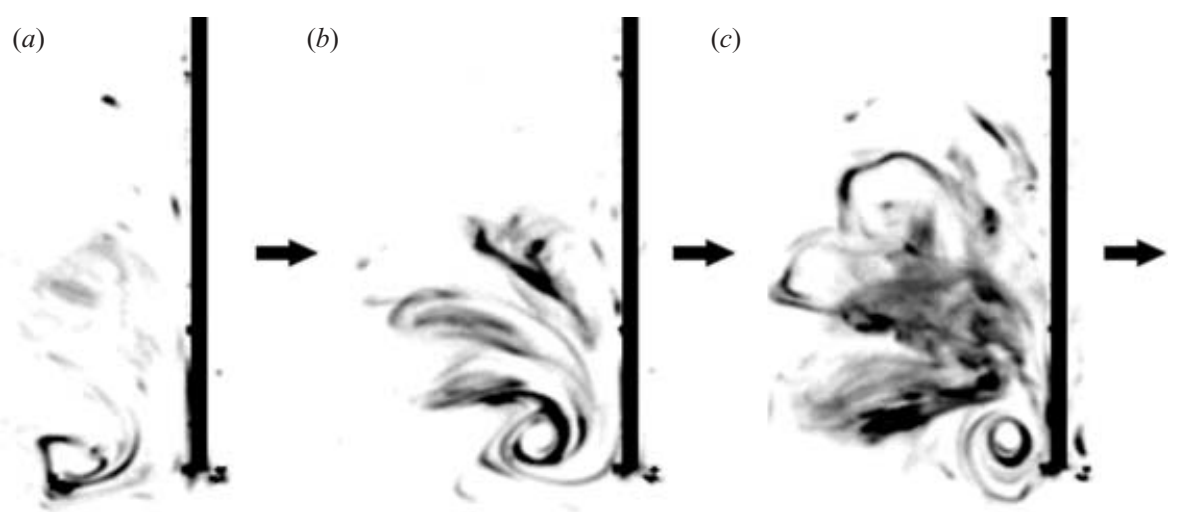

FIGURE 11. Mid-chord spanwise plane dye visualization; $A R=6, R e=3000$. (a) $t_{\text {ref }}$, about $3 c$ of travel; $(b) t_{\text {ref }}+0.27 \mathrm{~s} ;(c) t_{\text {ref }}+0.33 \mathrm{~s}$. Dye from the plate corners is convected into the mid-chord plane above the tip vortex.

\section{REFERENCES}

Batchelor, G. K. 1967 An Introduction to Fluid Dynamics, 1st edn. Cambridge University Press.

BirCH, J. \& Dickinson, M. H. 2001 Spanwise flow and the attachment of the leading-edge vortex on insect wings. Nature 412, 729-733.

ChUA, K., Lisoski, D., LeONARD, A. \& RoshKo, A. 1990 A numerical and experimental investigation of separated flow past an oscillating flat plate. ASME FED Intl Symp. on Nonsteady Fluid Dynamics 92, 455-464.

Dennis, S. C. R., Qiang, W., Coutanceau, M. \& Launay, J.-L. 1993 Viscous flow normal to a flat plate at moderate Reynolds numbers. J. Fluid Mech. 248, 605-635.

Dhawan, S. 1988 Bird Flight. Indian Academy of Sciences. 
Dickinson, M. H. \& GöTZ, K. G. 1993 Unsteady performance of model wings at low Reynolds number. J. Expl Biol. 174, 45-64.

Dickinson, M. H., Lehmann, F.-O. \& Sane, S. P. 1999 Wing rotation and the aerodynamic basis of insect flight. Science 284, 1954-1960.

Dudley, R. 2000 The Biomechanics of Insect Flight: Form, Function, Evolution. Princeton University Press.

Ellington, C. P. 1984 The aerodynamics of hovering insect flight. Phil. Trans. R. Soc. Lond. B305, $1-181$.

Ellington, C. P., van den Berg, C., Willmott, A. P. \& Thomas, A. L. R. 1996 Leading-edge vortices in insect flight. Nature 384, 626-630.

Koumoutsakos, P. \& Shiels, D. 1996 Simulations of the viscous flow normal to an impulsively started and uniformly accelerated flat plate. J. Fluid Mech. 328, 177-227.

Lian, Q.-X. \& HuANG, Z. 1989 Starting flows and structures of the starting vortex behind bluff bodies with sharp edges. Exps. Fluids 8, 95-103.

Lisoski, D. L. A. 1993 Nominally 2-dimensional flow about a normal flat plate. PhD thesis, California Institute of Technology.

Luchini, P. \& Tognaccini, R. 2002 The start-up vortex issuing from a semi-infinite flat plate. J. Fluid Mech. 455, 175-193.

Pullin, D. I. \& WANG, Z. J. 2004 Unsteady forces on an accelerating plate and application to hovering insect flight. J. Fluid Mech. 509, 1-21.

SARpKaYA, T. \& Kline, H. K. 1982 Impulsively-started flow about four types of bluff body. Trans. ASME I: J. Fluids Engng 104, 207-213.

Slaouti, A. \& Gerrard, J. H. 1981 An experimental investigation of the end effects on the wake of a circular cylinder towed through water at low Reynolds numbers. J. Fluid Mech. 112, 297-314.

WANG, Z. J. 2004 The role of drag in insect hovering. J. Expl Biol. 207, 4147-4155.

Westerweel, J., Dabiri, D. \& Gharib, M. 1997 The effect of a discrete window offset on the accuracy of cross correlation analysis of digital PIV recordings. Exps. Fluids 23, 20-28.

Willert, C. E. \& GHariB, M. 1991 Digital particle image velocimetry. Exps. Fluids 10, 181-193.

Willert, C. E. \& GHarib, M. 1997 The interaction of spatially modulated vortex pairs with free surfaces. J. Fluid Mech. 345, 227-250. 